\title{
QUASI-ELLIPTIC FUNCTIONS
}

\section{A.YA. KHRYSTIYANYN, DZ.V. LUKIVSKA}

\begin{abstract}
We study certain generalizations of elliptic functions, namely quasi-elliptic functions.

Let $p=e^{i \alpha}, q=e^{i \beta}, \alpha, \beta \in \mathbb{R}$. A meromorphic in $\mathbb{C}$ function $g$ is called quasi-elliptic if there exist $\omega_{1}, \omega_{2} \in \mathbb{C}^{*}, \operatorname{Im} \frac{\omega_{2}}{\omega_{1}}>0$, such that $g\left(u+\omega_{1}\right)=p g(u), g\left(u+\omega_{2}\right)=q g(u)$ for each $u \in \mathbb{C}$. In the case $\alpha=\beta=0 \bmod 2 \pi$ this is a classical theory of elliptic functions. A class of quasi-elliptic functions is denoted by $\mathcal{Q E}$. We show that the class $\mathcal{Q E}$ is nontrivial. For this class of functions we construct analogues $\wp_{\alpha \beta}, \zeta_{\alpha \beta}$ of $\wp$ and $\zeta$ Weierstrass functions. Moreover, these analogues are in fact the generalizations of the classical $\wp$ and $\zeta$ functions in such a way that the latter can be found among the former by letting $\alpha=0$ and $\beta=0$. We also study an analogue of the Weierstrass $\sigma$ function and establish connections between this function and $\wp_{\alpha \beta}$ as well as $\zeta_{\alpha \beta}$.

Let $q, p \in \mathbb{C}^{*},|q|<1$. A meromorphic in $\mathbb{C}^{*}$ function $f$ is said to be $p$-loxodromic of multiplicator $q$ if for each $z \in \mathbb{C}^{*} f(q z)=p f(z)$. We obtain telations between quasi-elliptic and $p$-loxodromic functions.
\end{abstract}

Keywords: quasi-elliptic function, the Weierstrass $\wp$-function, the Weierstrass $\zeta$-function, the Weierstrass $\sigma$-function, $p$-loxodromic function.

Mathematics subject classification: 30D30

\section{INTRODUCTION}

Denote $\mathbb{C}^{*}=\mathbb{C} \backslash\{0\}$. A meromorphic in $\mathbb{C}$ function $g$ is called elliptic [1] if there exist $\omega_{1}, \omega_{2} \in \mathbb{C}^{*}$ such that $\operatorname{Im} \frac{\omega_{2}}{\omega_{1}}>0$ and for each $u \in \mathbb{C}$

$$
g\left(u+\omega_{1}\right)=g(u), \quad g\left(u+\omega_{2}\right)=g(u) .
$$

The theory of elliptic functions was developed by K. Jacobi, N. Abel, A. Legendre, K. Weierstrass. The following definition was introduced by A. Kondratyuk.

Definition 1. 2] A meromorphic in $\mathbb{C}$ function $f$ is said to be modulo-elliptic if there exist $\omega_{1}, \omega_{2} \in \mathbb{C}^{*}$ such that $\operatorname{Im} \frac{\omega_{2}}{\omega_{1}}>0$ and for each $u \in \mathbb{C}$

$$
\left|f\left(u+\omega_{1}\right)\right|=|f(u)|, \quad\left|f\left(u+\omega_{2}\right)\right|=|f(u)| .
$$

Consider the first of these identities

$$
\left|f\left(u+\omega_{1}\right)\right|=|f(u)|, \quad u \in \mathbb{C} .
$$

If $f(u) \neq 0$ and $f(u) \neq \infty$, we can divide (1) by $|f(u)|$ to obtain

$$
\left|\frac{f\left(u+\omega_{1}\right)}{f(u)}\right|=1 \text {. }
$$

A.Ya. KhrystiYanyn, Dz.V. Lukivska, Quasi-elliptic functions.

(C)Khrystiyanyn A.Ya., Lukivska Dz.V. 2017.

Submitted September 27, 2016. 
The function $g(u)=\frac{f\left(u+\omega_{1}\right)}{f(u)}$ is meromorphic in $\mathbb{C}$. It follows from 22 that the function $g$ is holomorphic and bounded in $\mathbb{C}$ except for a set of the zeros and poles of $f$. Since $g$ is bounded, these points are removable, and relation (2) implies

$$
\forall u \in \mathbb{C}: \quad|g(u)|=1 .
$$

By the Liouville theorem $g$ is constant and the latter identity implies the existence of $\alpha \in \mathbb{R}$ such that $g(u)=e^{i \alpha}$. This means that

$$
\forall u \in \mathbb{C}: \quad f\left(u+\omega_{1}\right)=e^{i \alpha} f(u) .
$$

In the same way as above, we conclude that there exists $\beta \in \mathbb{R}$ such that

$$
\forall u \in \mathbb{C}: \quad f\left(u+\omega_{2}\right)=e^{i \beta} f(u) .
$$

We consider separately the following cases:

(i) $\alpha=\beta=0 \bmod 2 \pi$;

(ii) $\alpha=0 \bmod 2 \pi, \beta \neq 0 \bmod 2 \pi($ or $\alpha \neq 0 \bmod 2 \pi, \beta=0 \bmod 2 \pi)$;

(iii) $\alpha \neq 0 \bmod 2 \pi, \beta \neq 0 \bmod 2 \pi$.

In the first case we obtain the classical theory of elliptic functions including the famous Weierstrass $\wp$-function

$$
\wp(u)=\frac{1}{u^{2}}+\sum_{\omega \neq 0}\left(\frac{1}{(u-\omega)^{2}}-\frac{1}{\omega^{2}}\right), \quad \omega=m \omega_{1}+n \omega_{2}, \quad m, n \in \mathbb{Z} .
$$

The Weierstrass $\wp$-function is elliptic [1] with periods $\omega_{1}, \omega_{2}$. The representations for classical Weierstrass $\zeta$ and $\sigma$ functions are well-known [1], 3]:

$$
\begin{aligned}
& \zeta(u)=\frac{1}{u}+\sum_{\omega \neq 0}\left(\frac{1}{u-\omega}+\frac{1}{\omega}+\frac{u}{\omega^{2}}\right), \quad \omega=m \omega_{1}+n \omega_{2}, \quad m, n \in \mathbb{Z} . \\
& \sigma(u)=u \prod_{\omega \neq 0}\left(1-\frac{u}{\omega}\right) e^{\frac{u}{\omega}+\frac{u^{2}}{2 \omega^{2}}}, \quad \omega=m \omega_{1}+n \omega_{2}, \quad m, n \in \mathbb{Z} .
\end{aligned}
$$

We also observe that the following identities

$$
\wp(u)=-\zeta^{\prime}(u), \quad \zeta(u)=\frac{\sigma^{\prime}(u)}{\sigma(u)}, \quad \wp(u)=-\left(\frac{\sigma^{\prime}(u)}{\sigma(u)}\right)^{\prime} .
$$

hold true. We note that each elliptic function can be representedby using (3), (4), (5) (see [3]). In other words, these functions play an important role in representations of elliptic functions.

In the second case we obtain so-called $p$-elliptic functions.

Definition 2. [4] Let $p=e^{i \beta}$. A meromorphic in $\mathbb{C}$ function $g$ is called p-elliptic if there exist $\omega_{1}, \omega_{2} \in \mathbb{C}^{*}$ such that $\operatorname{Im} \frac{\omega_{2}}{\omega_{1}}>0$ and for each $u \in \mathbb{C}$

$$
g\left(u+\omega_{1}\right)=g(u), \quad g\left(u+\omega_{2}\right)=p g(u) .
$$

This case was studied in [6].

The aim of this article is to consider the third case. This is a generalization of elliptic functions in some sense as the following definition says.

Definition 3. Let $p=e^{i \alpha}, q=e^{i \beta}$. A meromorphic in $\mathbb{C}$ function $g$ is called quasi-elliptic if there exist $\omega_{1}, \omega_{2} \in \mathbb{C}^{*}, \operatorname{Im} \frac{\omega_{2}}{\omega_{1}}>0$, such that for each $u \in \mathbb{C}$

$$
g\left(u+\omega_{1}\right)=p g(u), \quad g\left(u+\omega_{2}\right)=q g(u) .
$$


We denote the class of quasi-elliptic functions by $\mathcal{Q E}$.

Let $\omega=m \omega_{1}+n \omega_{2}, m, n \in \mathbb{Z}$. If $f \in \mathcal{Q E}$, Definition 3 implies

$$
g(u+\omega)=p^{m} q^{n} g(u) .
$$

If $p=1$ and $q=1$ in Definition 3, we obtain classic elliptic function. If $p=1$ or $q=1$ in Definition 3, we obtain $p$-elliptic function.

Remark 1. There is one special case when Definition 3 still gives an elliptic function. Namely, if $p=e^{i \alpha}, q=e^{i \beta}$, where $\alpha, \beta \in 2 \pi \mathbb{Q}$, then

$$
f\left(u+l \omega_{1}\right)=f(u), \quad f\left(u+l \omega_{2}\right)=f(u),
$$

where $l$ is the least common denominator of $\frac{\alpha}{2 \pi}$ and $\frac{\beta}{2 \pi}$.

Indeed, if $\alpha=2 \pi \frac{a}{b}$, using Definition 3, we have

$$
f\left(u+l \omega_{1}\right)=f\left(u+(l-1) \omega_{1}\right) e^{i 2 \pi \frac{a}{b}}=\cdots=f(u) e^{i 2 \pi \frac{a l}{b}}=f(u) .
$$

The same conclusion can be made for $\beta$.

Remark 2. The class $\mathcal{Q E}$ of quasi-elliptic functions is not trivial. For example, consider the function

$$
f(u)=\sum_{\omega \neq 0} \frac{e^{i m \alpha} e^{i n \beta}}{(u-\omega)^{3}}, \quad \omega=m \omega_{1}+n \omega_{2}, \quad m, n \in \mathbb{Z} .
$$

Consider a compact subset $K$ from $\mathbb{C}$. Since ([1], 3])

$$
\sum_{\omega \neq 0} \frac{1}{|\omega|^{3}}<+\infty
$$

we obtain that the series in the right hand side of (6), or at least its remainder, is uniformly convergent on $K$. Therefore $f$ is meromorphic in $\mathbb{C}$, and we have for each $u \in \mathbb{C}$

$$
f\left(u+\omega_{1}\right)=e^{i \alpha} \sum_{m, n \in \mathbb{Z}} \frac{e^{i(m-1) \alpha} e^{i n \beta}}{\left(u-(m-1) \omega_{1}-n \omega_{2}\right)^{3}}=e^{i \alpha} f(u) .
$$

In the same way, for each $u \in \mathbb{C}$ we obtain $f\left(u+\omega_{2}\right)=e^{i \beta} f(u)$.

Our main aim is to construct a quasi-elliptic function $\wp_{\alpha \beta}$ being an analogue of $\wp(u)$ and also to construct corresponding analogues of $\zeta$ and $\sigma$ functions.

\section{Generalization of the Weierstrass $\wp$-FUnCtion}

Let $p=e^{i \alpha}, q=e^{i \beta}$. Consider the function

$$
G_{\alpha \beta}(u)=\frac{1}{u^{2}}+\sum_{\omega \neq 0}\left(\frac{1}{(u-\omega)^{2}}-\frac{1}{\omega^{2}}\right) e^{i(m \alpha+n \beta)},
$$

where $\omega_{1}, \omega_{2} \in \mathbb{C}, \operatorname{Im} \frac{\omega_{2}}{\omega_{1}}>0, \omega=m \omega_{1}+n \omega_{2}, m, n \in \mathbb{Z}$. Similarly, in view of (7), as in the case of the series from $(6)$, we obtain that $G_{\alpha \beta}$ is meromorphic in $\mathbb{C}$.

It is obvious that, $G_{00}$ coincides with the classical Weierstrass function $\wp$.

Consider the case $\alpha \neq 0 \bmod 2 \pi$ and $\beta \neq 0 \bmod 2 \pi$, that is, $p \neq 1$ and $q \neq 1$. 
Theorem 1. A function of the form

$$
\wp_{\alpha \beta}(u)=G_{\alpha \beta}(u)+C_{\alpha \beta},
$$

where

$$
C_{\alpha \beta}=\frac{G_{\alpha \beta}\left(\frac{\omega_{1}}{2}\right)-e^{i \alpha} G_{\alpha \beta}\left(-\frac{\omega_{1}}{2}\right)}{e^{i \alpha}-1}=\frac{G_{\alpha \beta}\left(\frac{\omega_{2}}{2}\right)-e^{i \beta} G_{\alpha \beta}\left(-\frac{\omega_{2}}{2}\right)}{e^{i \beta}-1}
$$

belongs to $\mathcal{Q E}$ with $p=e^{i \alpha}, q=e^{i \beta}$.

Proof. Consider the function $G_{\alpha \beta}$. We shall show that there exists a unique constant $C_{\alpha \beta}$ such that $\left(G_{\alpha \beta}(u)+C_{\alpha \beta}\right) \in \mathcal{Q E}$, that is

$$
\begin{aligned}
& G_{\alpha \beta}\left(u+\omega_{1}\right)+C_{\alpha \beta}=e^{i \alpha}\left(G_{\alpha \beta}+C_{\alpha \beta}\right), \\
& G_{\alpha \beta}\left(u+\omega_{2}\right)+C_{\alpha \beta}=e^{i \beta}\left(G_{\alpha \beta}+C_{\alpha \beta}\right) .
\end{aligned}
$$

These properties are called multi $p$-periodicity with the period $\omega_{1}$ and multi $q$-periodicity with the period $\omega_{2}$, respectively.

Let us consider the derivative of $G_{\alpha \beta}$ :

$$
G_{\alpha \beta}^{\prime}(u)=-2 \sum_{\omega} \frac{e^{i(m \alpha+n \beta)}}{(u-\omega)^{3}} .
$$

We have:

$$
\begin{aligned}
G_{\alpha \beta}^{\prime}\left(u+\omega_{1}\right) & =-2 \sum_{m, n \in \mathbb{Z}} \frac{e^{i(m \alpha+n \beta)}}{\left(u+\omega_{1}-m \omega_{1}-n \omega_{2}\right)^{3}}=-2 \sum_{m, n \in \mathbb{Z}} \frac{e^{i(m \alpha+n \beta)}}{\left(u-(m-1) \omega_{1}-n \omega_{2}\right)^{3}} \\
& =-2 e^{i \alpha} \sum_{m, n \in \mathbb{Z}} \frac{e^{i((m-1) \alpha+n \beta)}}{\left(u-(m-1) \omega_{1}-n \omega_{2}\right)^{3}}=e^{i \alpha} G_{\alpha \beta}^{\prime}(u) .
\end{aligned}
$$

Hence, we obtain

$$
G_{\alpha \beta}^{\prime}\left(u+\omega_{1}\right)-e^{i \alpha} G_{\alpha \beta}^{\prime}(u)=0 .
$$

We note that for each $C \in \mathbb{C}$, the function $\left(G_{\alpha \beta}+C\right)$ satisfies $(9)$. Let

$$
C=C_{\alpha \beta}=\frac{G_{\alpha \beta}\left(\frac{\omega_{1}}{2}\right)-e^{i \alpha} G_{\alpha \beta}\left(-\frac{\omega_{1}}{2}\right)}{e^{i \alpha}-1} .
$$

Then relation (9) implies

$$
G_{\alpha \beta}\left(u+\omega_{1}\right)+C_{\alpha \beta}-e^{i \alpha}\left(G_{\alpha \beta}+C_{\alpha \beta}\right)=A,
$$

where $A$ is a constant. If we let $u=-\frac{\omega_{1}}{2}$, it is easy to obtain that

$$
G_{\alpha \beta}\left(\frac{\omega_{1}}{2}\right)-e^{i \alpha} G_{\alpha \beta}\left(-\frac{\omega_{1}}{2}\right)+\left(1-e^{i \alpha}\right) C_{\alpha \beta}=A .
$$

Taking into consideration the choice of $C_{\alpha \beta}$ by formula $(10)$, we get $A=0$. Therefore, we have

$$
G_{\alpha \beta}\left(u+\omega_{1}\right)+C_{\alpha \beta}=e^{i \alpha}\left(G_{\alpha \beta}+C_{\alpha \beta}\right),
$$

that is, we have shown that the function $\left(G_{\alpha \beta}+C_{\alpha \beta}\right)$ is multi $p$-periodic of period $\omega_{1}$.

It remains to prove the uniqueness of $C_{\alpha \beta}$. Suppose that there exists a constant $C$ different from $C_{\alpha \beta}$ such that the function $\left(G_{\alpha \beta}+C\right)$ is multi $p$-periodic of period $\omega_{1}$, too. Then we get

$$
G_{\alpha \beta}\left(u+\omega_{1}\right)+C=e^{i \alpha}\left(G_{\alpha \beta}(u)+C\right) .
$$

Deducting this identity from (11), we obtain

$$
C-C_{\alpha \beta}=e^{i \alpha}\left(C-C_{\alpha \beta}\right) .
$$


Since $\alpha \neq 0 \bmod 2 \pi$, we get $C=C_{\alpha \beta}$.

In the same way, for the period $\omega_{2}$ we have

$$
G_{\alpha \beta}\left(u+\omega_{2}\right)+C_{\alpha \beta}=e^{i \beta}\left(G_{\alpha \beta}(u)+C_{\alpha \beta}\right)+B,
$$

where $B$ is some constant.

Let us find $B$. Using identities (11) and (12), we obtain

$$
\begin{aligned}
G_{\alpha \beta}\left(u+\omega_{1}+\omega_{2}\right)+C_{\alpha \beta} & =e^{i \beta}\left(G_{\alpha \beta}\left(u+\omega_{1}\right)+C_{\alpha \beta}\right)+B \\
& =e^{i \beta}\left(e^{i \alpha}\left(G_{\alpha \beta}(u)+C_{\alpha \beta}\right)\right)+B \\
& =e^{i(\alpha+\beta)}\left(G_{\alpha \beta}(u)+C_{\alpha \beta}\right)+B
\end{aligned}
$$

and

$$
\begin{aligned}
G_{\alpha \beta}\left(u+\omega_{1}+\omega_{2}\right)+C_{\alpha \beta} & =e^{i \alpha}\left(G_{\alpha \beta}\left(u+\omega_{2}\right)+C_{\alpha \beta}\right) \\
& =e^{i \alpha}\left(e^{i \beta}\left(G_{\alpha \beta}(u)+C_{\alpha \beta}\right)+B\right) \\
& =e^{i(\alpha+\beta)}\left(G_{\alpha \beta}(u)+C_{\alpha \beta}\right)+B e^{i \alpha} .
\end{aligned}
$$

Comparing the right hand sides of these relations, we get $B=B e^{i \alpha}$. Since $\alpha \neq 0 \bmod 2 \pi$, the previous identity implies that $B=0$. Therefore,

$$
G_{\alpha \beta}\left(u+\omega_{2}\right)+C_{\alpha \beta}=e^{i \beta}\left(G_{\alpha \beta}(u)+C_{\alpha \beta}\right) .
$$

Hence, the function $G_{\alpha \beta}$ is multi $p$-periodic with the period $\omega_{1}$ and is multi $q$-periodic with period $\omega_{2}$, respectively.

It is easy to see that $C_{\alpha \beta}$ can be also expressed as

$$
C_{\alpha \beta}=\frac{G_{\alpha \beta}\left(\frac{\omega_{2}}{2}\right)-e^{i \beta} G_{\alpha \beta}\left(-\frac{\omega_{2}}{2}\right)}{e^{i \beta}-1} .
$$

Definition 4. A function of the form

$$
\wp_{\alpha \beta}(u)=G_{\alpha \beta}(u)+C_{\alpha \beta}=\frac{1}{u^{2}}+\sum_{\omega \neq 0}\left(\frac{1}{(u-\omega)^{2}}-\frac{1}{\omega^{2}}\right) e^{i(m \alpha+n \beta)}+C_{\alpha \beta},
$$

where

$$
C_{\alpha \beta}=\frac{G_{\alpha \beta}\left(\frac{\omega_{1}}{2}\right)-e^{i \alpha} G_{\alpha \beta}\left(-\frac{\omega_{1}}{2}\right)}{e^{i \alpha}-1}=\frac{G_{\alpha \beta}\left(\frac{\omega_{2}}{2}\right)-e^{i \beta} G_{\alpha \beta}\left(-\frac{\omega_{2}}{2}\right)}{e^{i \beta}-1}
$$

is called the generalized Weierstrass $\wp$-function.

Remark 3. For the sake of completeness, in the case $p=q=1$, in other words, as $\alpha=\beta=0$ $\bmod 2 \pi$, we efine $C_{00}=0$. Then $\wp_{00}=\wp$.

\section{Generalization of Weierstrass $\zeta$ And $\sigma$ Functions}

Now we consider the function

$$
\zeta_{\alpha \beta}(u)=\frac{1}{u}+\sum_{\omega \neq 0}\left(\frac{1}{u-\omega}+\frac{1}{\omega}+\frac{u}{\omega^{2}}\right) e^{i(m \alpha+n \beta)},
$$

where $\omega_{1}, \omega_{2} \in \mathbb{C}, \operatorname{Im} \frac{\omega_{2}}{\omega_{1}}>0, \omega=m \omega_{1}+n \omega_{2}, m^{2}+n^{2} \neq 0, m, n \in \mathbb{Z}$.

Differentiating $\zeta_{\alpha \beta}$, we obtain $G_{\alpha \beta}(u)=-\zeta_{\alpha \beta}^{\prime}(u)$. Hence,

$$
\wp_{\alpha \beta}(u)=-\zeta_{\alpha \beta}^{\prime}(u)+C_{\alpha \beta} .
$$


We denote

$$
\chi_{m n}(u)=\left(\frac{1}{u-\omega}+\frac{1}{\omega}+\frac{u}{\omega^{2}}\right), \quad m^{2}+n^{2} \neq 0,
$$

and

$$
\chi_{00}(u)=\frac{1}{u}
$$

Then $\zeta_{\alpha \beta}$ can be rewritten as

$$
\zeta_{\alpha \beta}(u)=\sum_{m, n \in \mathbb{Z}} e^{i(m \alpha+n \beta)} \chi_{m n}(u)
$$

We observe that $\zeta_{00}$ coincides with the classical Weierstrass $\zeta$ function.

By $A^{*}$ we denote the plane $\mathbb{C}$ with radial slits from $\omega$ to $\infty$. Integrating $\chi_{m n}$ and $\chi_{00}$ along a path in $A^{*}$ connecting the points 0 and $u$, we obtain

$$
\int_{0}^{u} \chi_{m n}(t) d t=\log \left(1-\frac{u}{\omega}\right)+\frac{u}{\omega}+\frac{u^{2}}{2 \omega^{2}}, \quad m^{2}+n^{2} \neq 0
$$

and

$$
\int_{0}^{u} \chi_{00}(t) d t=\log u
$$

We consider entire functions

$$
\sigma_{m n}(u)=\left(1-\frac{u}{\omega}\right) e^{\frac{u}{\omega}+\frac{u^{2}}{2 \omega^{2}}}, \quad m^{2}+n^{2} \neq 0
$$

and we let

$$
\sigma_{00}(u)=u
$$

Employing these functions, we can rewrite (14) as

$$
\int_{0}^{u} \chi_{m n}(t) d t=\log \sigma_{m n}(u), \quad m, n \in \mathbb{Z} .
$$

Differentiating this identity and using the definitions of $\chi_{00}$ and $\sigma_{00}$, we get

$$
\forall m, n \in \mathbb{Z}: \quad \chi_{m n}(u)=\frac{\sigma_{m n}^{\prime}(u)}{\sigma_{m n}(u)} .
$$

Taking into consideration this representation for $\chi_{m n}$, we rewrite 13 as

$$
\zeta_{\alpha \beta}(u)=\sum_{m, n \in \mathbb{Z}} e^{i(m \alpha+n \beta)} \frac{\sigma_{m n}^{\prime}(u)}{\sigma_{m n}(u)} .
$$

Hence, $\wp_{\alpha \beta}$ can be rewritten as

$$
\wp_{\alpha \beta}(u)=C_{\alpha \beta}+\sum_{m, n \in \mathbb{Z}} e^{i(m \alpha+n \beta)} \frac{\left(\sigma_{m n}^{\prime}(u)\right)^{2}-\sigma_{m n}^{\prime \prime}(u) \sigma_{m n}(u)}{\sigma_{m n}^{2}(u)} .
$$

We note that if we consider the product $\prod_{m, n \in \mathbb{Z}} \sigma_{m n}(u)$, then we obtain the Weierstrass $\sigma$-function. 


\section{ConneCtion BetweEn p-LOXODROMIC AND QUASI-ELLIPTIC FUnCTIONS}

Let $q, p \in \mathbb{C}^{*},|q|<1$.

Definition 5. [5] A meromorphic in $\mathbb{C}^{*}$ function $f$ is said to be p-loxodromic with the multiplicator $q$ if $f(q z)=p f(z)$ for each $z \in \mathbb{C}^{*}$.

We denote by $\mathcal{L}_{q p}$ the class of $p$-loxodromic functions with the multiplicator $q$.

The case $p=1$ was studied earlier in the works of O. Rausenberger [7], G. Valiron [8] and Y. Hellegouarch [1]. In this case the function $f$ is called loxodromic.

Let $a_{1}=e^{2 \pi i \frac{\omega_{2}}{\omega_{1}}}, a_{2}=e^{2 \pi i \frac{\omega_{1}}{\omega_{2}}}$ and $f_{1} \in \mathcal{L}_{a_{1} q}, f_{2} \in \mathcal{L}_{a_{2} p}$. Then

$$
f_{1}\left(a_{1} z\right)=q f_{1}(z), \quad f_{2}\left(a_{2} z\right)=p f_{2}(z) .
$$

We define

Then $g \in \mathcal{Q E}$. Indeed,

$$
g(u):=f_{1}\left(e^{2 \pi i \frac{u}{\omega_{1}}}\right) f_{2}\left(e^{2 \pi i \frac{u}{\omega_{2}}}\right) .
$$

$$
\begin{aligned}
g\left(u+\omega_{1}\right) & =f_{1}\left(e^{2 \pi i \frac{u}{\omega_{1}}}\right) f_{2}\left(e^{2 \pi i \frac{u}{\omega_{2}}} e^{2 \pi i \frac{\omega_{1}}{\omega_{2}}}\right) \\
& =f_{1}\left(e^{2 \pi i \frac{u}{\omega_{1}}}\right) f_{2}\left(a_{2} e^{2 \pi i \frac{u}{\omega_{2}}}\right) \\
& =p f_{1}\left(e^{2 \pi i \frac{u}{\omega_{1}}}\right) f_{2}\left(e^{2 \pi i \frac{u}{\omega_{2}}}\right)=p g(u),
\end{aligned}
$$

and

$$
\begin{aligned}
g\left(u+\omega_{2}\right) & =f_{1}\left(e^{2 \pi i \frac{u}{\omega_{1}}} e^{2 \pi i \frac{\omega_{2}}{\omega_{1}}}\right) f_{2}\left(e^{2 \pi i \frac{u}{\omega_{2}}}\right) \\
& =f_{1}\left(a_{1} e^{2 \pi i \frac{u}{\omega_{1}}}\right) f_{2}\left(e^{2 \pi i \frac{u}{\omega_{2}}}\right) \\
& =q f_{1}\left(e^{2 \pi i \frac{u}{\omega_{1}}}\right) f_{2}\left(e^{2 \pi i \frac{u}{\omega_{2}}}\right)=q g(u) .
\end{aligned}
$$

Vice versa, let $g \in \mathcal{Q E}, p=1, q \neq 1$, that is

$$
g\left(u+\omega_{1}\right)=g(u), \quad g\left(u+\omega_{2}\right)=q g(u) .
$$

We denote

$$
f(z):=g\left(\frac{\omega_{1}}{2 i \pi} \log z\right) .
$$

The function $f$ is well-defined since $g$ admits the period $\omega_{1}$ and therefore, the substitution of $\log z$ by $\log z+2 \pi i k, k \in \mathbb{Z}$ does not change the value of $g$ in the right hand side of (16). In other words, here the composition of a multivalent mapping with a univalent one is a univalent function. Hence, if we let $a=e^{2 \pi i \frac{\omega_{2}}{\omega_{1}}}, \operatorname{Im} \frac{\omega_{2}}{\omega_{1}}>0$, we obtain

$$
\begin{aligned}
f(a z) & =g\left(\frac{\omega_{1}}{2 i \pi} \log (a z)\right)=g\left(\omega_{2}+\frac{\omega_{1}}{2 i \pi} \log z\right) \\
& =q g\left(\frac{\omega_{1}}{2 i \pi} \log z\right)=q f(z) .
\end{aligned}
$$

Thus, $f \in \mathcal{L}_{a q}$. The case $p \neq 1, q=1$ is similar. We let

$$
f(z):=g\left(\frac{\omega_{2}}{2 i \pi} \log z\right)
$$

and $a=e^{2 \pi i \frac{\omega_{1}}{\omega_{2}}}$. Then $f \in \mathcal{L}_{a p}$. Indeed,

$$
\begin{aligned}
f(a z) & =g\left(\frac{\omega_{2}}{2 i \pi} \log (a z)\right)=g\left(\omega_{1}+\frac{\omega_{2}}{2 i \pi} \log z\right) \\
& =p g\left(\frac{\omega_{2}}{2 i \pi} \log z\right)=p f(z) .
\end{aligned}
$$

In the case $p \neq 1, q \neq 1$ the functions $g\left(\frac{\omega_{k}}{2 i \pi} \log z\right)$ are multivalent, $k=1,2$. 


\section{REFERENCES}

1. Y. Hellegouarch. Invitation to the Mathematics of Fermat-Wiles. Academic Press, San Diego (2002).

2. V.S. Khoroshchak, A.A. Kondratyuk. Some steps to nonlinear analysis // in Book of Abstracts of "XVIII-th Conference on analytic functions and related topics". Chełm, Poland. 38-39 (2016).

3. A. Hurwitz, R. Courant. Function theory. Nauka, Moscow (1968). (in Russian).

4. V.S. Khoroshchak, A.A. Kondratyuk. Generalization of the Weierstrass $\wp$-function // in Book of Abstracts of Ukrainian Scientific Conference "Modern problems of probability theory and mathematical analysis", Vorokhta, Ukraine, 93 (2016) (in Ukrainian).

5. V.S. Khoroshchak, A.Ya. Khrystiyanyn, D.V. Lukivska. A class of Julia exceptional functions // Carpathian Math. Publ. 8:1, 172-180 (2016).

6. A.A. Kondratyuk, V.S. Khoroshchak, D.V. Lukivska. p-Elliptic functions // Visnyk Lviv Univ. Ser. Mech. Math. 81, 121-129 (2016).

7. O. Rausenberger. Lehrbuch der Theorie der Periodischen Functionen Einer variabeln. // Druck und Ferlag von B.G.Teubner, Leipzig (1884).

8. G. Valiron. Cours d'Analyse mathématique: Théorie des fonctions. Masson, Paris. (1948).

Andriy Yaroslavovych Khrystiyanyn,

Ivan Franko National University of Lviv,

Universytetska str., 1,

79000, Lviv, Ukraine

E-mail: khrystiyanyn@ukr.net

Dzvenyslava Volodymyrivna Lukivska,

Ivan Franko National University of Lviv,

Universytetska str., 1,

79000, Lviv, Ukraine

E-mail: d.lukivska@gmail.com 